\title{
The jamming transition and new percolation universality classes in particulate systems with attraction
}

\author{
Gregg Lois, Jerzy Blawzdziewicz, and Corey S. O'Hern \\ Department of Mechanical Engineering, Department of Physics, \\ Yale University, New Haven, Connecticut 06520-8284
}

\begin{abstract}
We numerically study the jamming transition in particulate systems with attraction by investigating their mechanical response at zero temperature. We find three regimes of mechanical behavior separated by two critical transitions - connectivity and rigidity percolation. The transitions belong to different universality classes than their lattice counterparts, due to force balance constraints. We also find that these transitions are unchanged at low temperatures and resemble gelation transitions in experiments on colloidal and silica gels.
\end{abstract}

The provocative conjecture that mechanical response at zero temperature is linked to slow dynamics at nonzero temperature in repulsive glassy systems has sparked tremendous interest in the jamming transition at "point J" 1]. At point J athermal, frictionless systems with finite-range repulsive interactions [2] undergo a single critical transition from an unjammed state with a vanishing static shear modulus to a jammed state with a non-zero static shear modulus as density is increased. At the transition, it is observed that the average number of particles participating in the connected network jumps discontinuously from 0 to $L^{d}$ (where $d$ is the spatial dimension and $L$ is the system size), which makes the transition first-order in the network order parameter. Recent work has indeed shown that properties of the jammed state are closely related to the slow dynamics in highly compressed repulsive glasses [3].

How does the jamming transition change for particulate systems with attraction? It is likely that attractive interactions will qualitatively change the nature of the jamming transition. For example, thermal systems with attraction can form repulsive glasses, but they can also form gels and attractive glasses [4]. Moreover, at zero temperature the mechanical properties of attractive granular materials and powders are quite different than those for dry granular media with purely repulsive interactions [5]. While the jamming phase diagram of Ref. [1] needs revision to include attraction, the close correspondence between mechanical properties at zero temperature and dynamics at non-zero temperature is robust. This has been demonstrated in experiments on attractive colloidal suspensions [ [6] where, as expected from the jamming phase diagram, gelation occurs upon increasing density, decreasing thermalization, and decreasing stress.

In this Letter, we explore the jamming transition in attractive particulate systems by studying their mechanical response at zero temperature. A central conclusion from our work is that repulsive jamming is fundamentally different than attractive jamming, even for an infinitesimal amount of attraction. Instead of a single first-order transition in the purely repulsive systems, we observe two second-order transitions in the attractive systems- connectivity and rigidity percolation. These two transitions separate three distinct types of mechanical response and exhibit critical exponents that differ from those measured in the connectivity and rigidity percolation transitions without force balance constraints. The transitions we observe at zero temperature are also present at small but finite temperatures and resemble gelation.

Simulation procedure We investigate the quasistatic compression of an attractive particulate system in two and three dimensions at zero temperature. The system consists of $N$ particles interacting via a pairwise, spherically symmetric potential, with a finite repulsive core and finite-range attraction. Simulations begin with a dilute collection of $N$ spherical particles of diameter $\sigma_{i}$ randomly placed in a cubic cell with periodic boundary conditions. In each simulation step the diameters of all particles are increased by a small factor and then the potential energy is minimized using a conjugate gradient method. The force $F\left(r_{i j}\right)$ between a pair of particles $i$ and $j$ depends on their separation $r_{i j}$ relative to the sum of their radii $\sigma_{i j}=\left(\sigma_{i}+\sigma_{j}\right) / 2$, and is plotted in the inset of Fig. 1(a). Since the overall scale $Y$ of the force is irrelevant at zero temperature, there are three independent parameters: the packing fraction $\phi$ (calculated using the location of the minimum in the force law), the range of the attraction $\ell$, and the minimum scaled force $-C$.

Mechanical Response At zero temperature, whether a particulate system with attractions is jammed depends on two important factors: a) does the system contain a percolating cluster and b) if so, does the percolating cluster possess any floppy modes? A floppy mode [7] is an infinitesimal deformation of the material that does not increase its energy. The number of floppy modes is equal to the number of non-trivial zero eigenvalues of the dynamical matrix for a given set of particle positions and radii [8]. If there are no non-trivial [9] zero eigenvalues, the potential energy increases with any deformation and all elastic constants are non-zero. We refer to this state as "jammed". If there is at least one non-trivial zero eigenvalue, the system is termed "unjammed". Note that this is a very strict definition of jamming.

It is impossible to have a jammed system that does not 
(a)

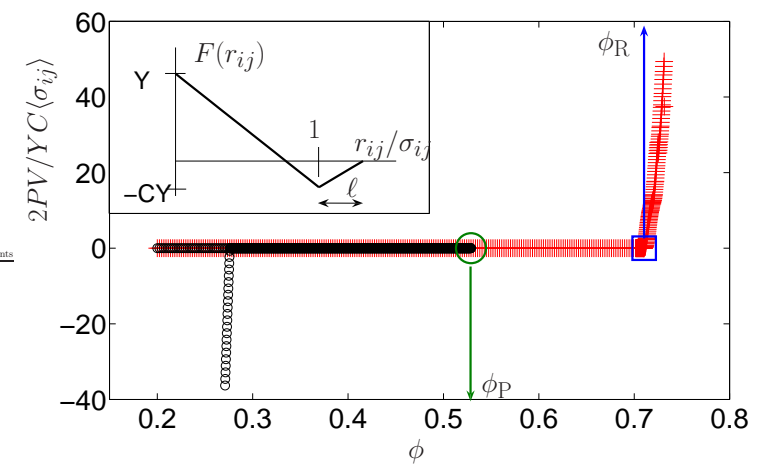

(b)

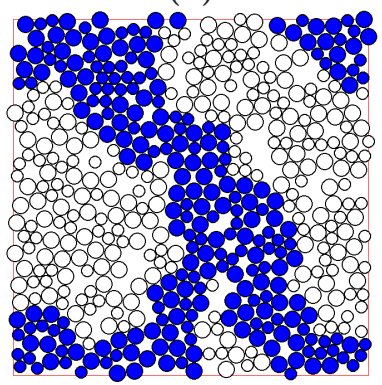

(d)

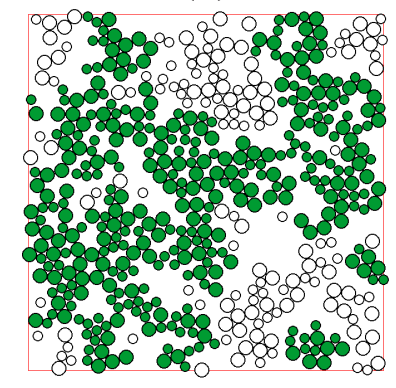

(c)

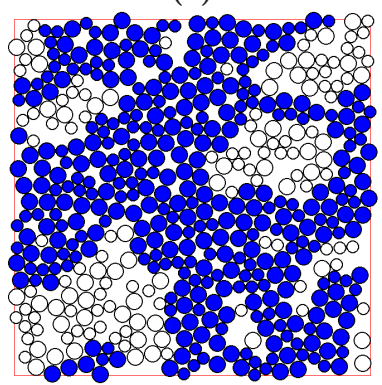

(e)

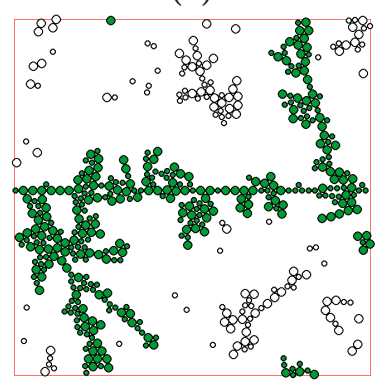

FIG. 1: (Color online). (a) The pressure $P$ vs. $\phi$ for two different compression procedures, which both begin at $P=0$ and $\phi=0.2$. Red crosses correspond to the jamming path, where the system is compressed until it becomes jammed at the onset of rigidity percolation $\phi_{\mathrm{R}}$. Black circles correspond to the connectivity percolation path, where the system is compressed to the onset of connectivity percolation $\phi_{\mathrm{P}}$ and then decompressed. The inset shows the central force law used in our simulations. (b) and (c) Screenshots at $\phi_{\mathrm{R}}$ for two different systems. The blue-colored particles comprise the percolating backbone. (d) Screenshot at $\phi_{\mathrm{P}}$, with particles in the percolating cluster colored green. (e) Screenshot of the system pictured in (d) after decompression, where the percolating structure is stretched to its fullest extent.

percolate, which leaves three distinct possibilities for its mechanical state: (1) nonpercolated, (2) percolated but unjammed, and (3) jammed. In repulsive systems only (1) and (3) occur and they are separated by the firstorder jamming transition at point J. In the attractive particulate systems investigated here, all three mechanical states occur. A second-order connectivity percolation transition separates (1) and (2), and a second-order rigidity percolation transition separates (2) and (3).

Properties of the three mechanical states are illustrated

by measuring the pressure $P$. In Fig. 1(a) we plot $P$ vs. $\phi$ for two compression procedures in a $d=2$ system with $N=512, C=10^{-2}$, and $\ell=0$. In the first procedure, marked by red crosses, the system is compressed until it jams at $\phi_{\mathrm{R}}$, after which the pressure becomes nonzero. At $\phi_{\mathrm{R}}$ a percolating backbone forms with no floppy modes, also known as rigidity percolation [16]. Screenshots of the system at $\phi_{\mathrm{R}}$ are shown in Figs. $1(\mathrm{~b})$ and (c). Note that while there are no floppy modes in the percolating backbone, there are trivial floppy modes in the system involving particles not in the backbone [10].

In the second procedure, marked by black circles, the same initial system is compressed until a percolating network forms at $\phi_{\mathrm{P}}$, as illustrated in Fig. I(d). Then the system is decompressed. We find that upon decompression the pressure remains zero until a very low packing fraction. At this point the network is stretched to its fullest extent with no tension, as illustrated in Fig. 1(e). Further decompression causes tension $(P<0)$ until the cluster becomes unstable, breaks, and $P=0$. The formation of a percolating cluster gives rise to novel mechanical behavior - infinitesimal deformations do not alter the potential energy but large deformations do. Thus, the mechanical response of the percolated but unjammed state cannot be described by linear elasticity.

The mechanical behavior described above is generic. For all compaction procedures we investigated, connectivity percolation and rigidity percolation are distinct transitions, with $\phi_{\mathrm{P}}<\phi_{\mathrm{R}}$. This occurs despite the fact that the mechanical response of the system is highly history dependent. Since breaking a contact between particles has an energy cost, contacts gained upon compression are not necessarily lost upon decompression and we observe that the number of contacts per particle $z$ never decreases when $P=0$. Because contacts are not broken, clusters of particles grow, or coarsen. This leads to work-hardening and lowers $\phi_{\mathrm{P}}$ and $\phi_{\mathrm{R}}$.

Connectivity Percolation Connectivity percolation occurs when a cluster of interacting particles first spans the system. The transition is characterized by three independent critical exponents, unless the hyperscaling assumption holds, in which case the number is two 11]. The choice of three independent exponents from a larger number of measurable exponents is arbitrary. We choose to focus on (1) the correlation length exponent $\nu$ which quantifies the divergence of the linear extent $\xi$ of the largest non-percolating cluster $\xi \propto\left|\phi-\phi_{\mathrm{P}}\right|^{-\nu}$, (2) the cluster-statistics exponent $\tau$ which characterizes the number $n_{s}$ of clusters with $s$ particles at the percolation transition $n_{s}\left(\phi_{\mathrm{P}}\right) \propto s^{-\tau}$, and (3) the fractal dimension $D$ at $\phi_{\mathrm{P}}$. The hyperscaling relation $D(\tau-1)=d$ holds if $\xi$ is the only relevant length scale in the system.

The critical exponents of the connectivity percolation transition have been determined for lattices 11] and for continuum particulate systems without interparticle forces [12, 13]. The critical exponents, which are listed 

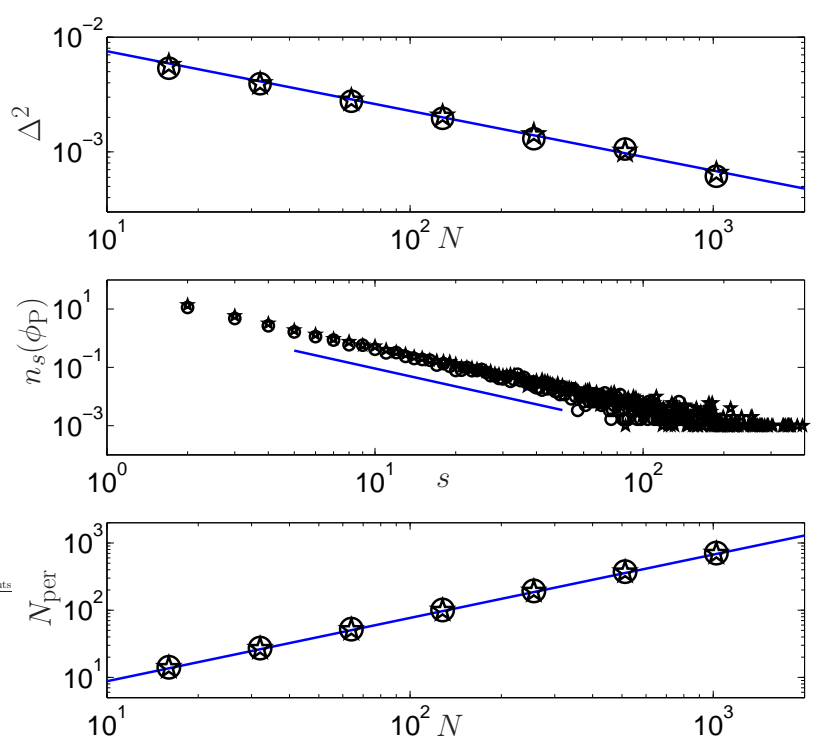

FIG. 2: Measurement of the connectivity percolation exponents in $d=2$. Circles (stars) correspond to monodisperse (bidisperse) distributions. (a) The square width of connectivity percolation thresholds $\Delta^{2}$ as a function of system size $N$. The slope gives $-2 / d \nu$. (b) The number of clusters of size $s$ at $\phi_{\mathrm{P}}$. The slope gives $-\tau$. (c) The average number of particles in the percolation cluster $N_{\mathrm{per}}$ at $\phi_{\mathrm{P}}$, as a function of system size $N$. The slope gives $D / d$. The best fit slopes determined from our analysis are included in each plot.

in Table【, are identical for lattice and continuum percolation and do not depend on the lattice type. In static particulate systems, force balance is crucial and has not been included in previous continuum connectivity percolation studies. In what follows we measure the independent connectivity percolation exponents, test the hyperscaling hypothesis, and compare our results to lattice connectivity percolation.

In Fig. 2 we show the data used to determine the connectivity percolation exponents in $d=2$ for both monodisperse and bidisperse 14 systems. Every data point is averaged over 5000 trials, each consisting of an initially random, dilute state that is subsequently compressed to the percolation onset at $\phi_{\mathrm{P}}$. The correlation length exponent $\nu$ is measured using the standard finite size scaling analysis [11], where the root mean square width $\Delta$ of the $\phi_{\mathrm{P}}$ distribution is related to the system size $L$ by $\Delta \propto L^{1 / \nu}$. In Fig. 2(a) we plot $\Delta^{2}$ versus $N$ and observe a power law behavior with an exponent of $-2 / d \nu$. This gives $\nu=1.92 \pm 0.03$, which is larger than the value of $4 / 3$ for lattice connectivity percolation. Next, we determine the exponent $\tau$ by directly measuring the cluster-size distribution. In Fig. 2(b) we plot $n_{s}\left(\phi_{\mathrm{P}}\right)$ for a system with 1024 particles and find that $n_{s}\left(\phi_{\mathrm{P}}\right) \propto s^{-\tau}$ with $\tau=2.04 \pm 0.04$. This is consistent with the lattice result of 187/91. Finally, in Fig. 2(c) we plot the average number of particles in the percolating cluster $N_{\text {per }}$ as a function of $N$ at $\phi_{\mathrm{P}}$. We find that $N_{\text {per }} \propto N^{D / d}$, where $D=1.88 \pm 0.04$ is the fractal di-

\begin{tabular}{lccc}
\hline \hline System & $\nu$ & $\tau$ & $D$ \\
\hline present work $d=2$ & $1.92 \pm 0.03$ & $2.04 \pm 0.04$ & $1.88 \pm 0.04$ \\
lattice $d=2[11]$ & $4 / 3(1.33)$ & $187 / 91(2.05)$ & $91 / 48(1.90)$ \\
continuum $d=2[12]$ & $1.34 \pm 0.02$ & $2.0 \pm 0.1$ & $1.91 \pm 0.04$ \\
present work $d=3$ & $1.10 \pm 0.03$ & $2.20 \pm 0.03$ & $2.56 \pm 0.06$ \\
lattice $d=3[11]$ & 0.88 & 2.18 & 2.53 \\
continuum $d=3[13]$ & $0.94 \pm 0.20$ & $2.22 \pm 0.08$ & $2.21 \pm 0.56$ \\
\hline \hline
\end{tabular}

TABLE I: Critical exponents for connectivity percolation.

mension at percolation. This is also consistent with the lattice result of $91 / 48$.

A similar analysis has been applied to $d=3$; the measured exponents in $d=2$ and 3 are collected in Tableप. In both cases $D$ and $\tau$ are consistent with lattice and continuum connectivity percolation, while $\nu$ is larger than the classical value. The hyperscaling relation $D(\tau-1)=d$ is consistent with our results.

We have tested the robustness of the measured exponents by altering key parameters in the force law and by varying the preparation algorithm. First, we varied the minimum force $C$ over several orders of magnitude from $10^{-4}$ to $10^{-1}$ and the range of the attraction $\ell$ from 0 to 4 . Second, instead of compressing, we tested for percolation by quenching to zero temperature at constant volume. Third, we redefined the connectivity percolation transition to require percolation in the $x-, y-$ or $z$-directions or all directions at the same time. In all of these cases the connectivity percolation exponents remain the same as those listed in Table I. We also expect that the measured exponents do not change if the system is allowed to coarsen from successive decompressions and compressions or other methods. If the system coarsens before it percolates, the length scale over which structure is affected has a finite extent (equal to the size of the largest cluster after coarsening). Near percolation $\xi$ is much larger than this length scale and coarsening is irrelevant. However, we were not able to directly verify this conjecture because of insufficient system size.

Based on these, considerations we conclude that the measured exponents (shown in Table \) are universal for attractive, frictionless particulate systems. Thus we have identified a new universality class - connectivity percolation with force balance - that is different from connectivity percolation in the absence of forces. The universality class changes abruptly when $Y>0$ (emergence of excluded volume) and $C>0$ (emergence of attraction).

Rigidity Percolation The rigidity percolation transition at $\phi_{\mathrm{R}}$, which separates the percolated-unjammed and jammed states, occurs when a set of particles with no floppy modes has percolated. We refer to this set as the backbone. In simulations we identify backbone particles as those with at least one non-zero contact force (stressed particles). Below $\phi_{\mathrm{R}}$ we always observe that no particles are stressed and above $\phi_{\mathrm{R}}$ a percolating cluster of stressed particles with no floppy modes exists. Figs. 1(b) and (c) 


\begin{tabular}{lccc}
\hline \hline System & $\nu$ & $\tau$ & $D_{b}$ \\
\hline present work $d=2$ & $3.12 \pm 0.02$ & $2.08 \pm 0.06$ & $1.77 \pm 0.05$ \\
lattice $d=2[16]$ & $1.17 \pm 0.02$ & - & $1.78 \pm 0.02$ \\
present work $d=3$ & $0.92 \pm 0.01$ & $2.31 \pm 0.18$ & $2.58 \pm 0.16$ \\
\hline \hline
\end{tabular}

TABLE II: Critical exponents for rigidity percolation.

are screenshots of two systems at rigidity percolation. We have also used $P>0$ to define the onset of rigidity percolation, and obtain identical results [15].

Similar to connectivity percolation, there are three independent critical exponents in rigidity percolation, and we choose $\xi, \tau$ and $D_{b}$ (backbone fractal dimension) to characterize our system. Numerical values of these exponents are listed in Table II along with the corresponding lattice results. Rigidity percolation on a lattice has been studied in $d=2$ [16] and $d=3$ [17]. In the $d=2$ lattice studies the transition is second-order but only the exponents $D_{b}$ and $\nu$ have been measured. Our measurement of $\nu$ differs from the lattice value, while $D_{b}$ remains the same. In the $d=3$ lattice studies, the order of the transition depends on lattice type, which makes comparison with our off-lattice results difficult. Hyperscaling is consistent with our results both in $d=2$ and 3 .

We have also measured the fractal dimension $D$ of the entire percolating cluster, and we find that $D=d$. Therefore, while the rigidity percolation transition is second order in the backbone particles, it might appear to be a discontinuous first-order transition if the entire percolating cluster is mistaken for the rigid percolating cluster.

Extension to finite temperature In this letter, we have considered attractive particulate systems at zero temperature. We are currently investigating the effects of thermal fluctuations. When $T>0$, contact formation due to particle or cluster diffusion and contact breaking due to thermal activation become relevant. If $T$ is much smaller than the energy needed to break a contact and the diffusion length scale is small, we expect the $T=0$ results to hold. This has been verified for connectivity percolation in our preliminary studies of these systems undergoing Brownian dynamics.

Experimental verification of our predictions can also be found in the literature. First, in Ref. [18], experiments on colloidal gels demonstrate that the connectivity percolation transition (associated with gelation) occurs at a lower density than the rigidity percolation transition (associated with arrested dynamics at all length scales). This is consistent with our observation that connectivity and rigidity percolation occur at different densities. Second, the critical exponents for gelation have been measured as $\nu=1.17 \pm 0.10$ [19], $\tau=2.15 \pm 0.05$ [20] in colloidal suspensions, and $\nu=1.22 \pm 0.16, \tau=2.26 \pm 0.08$ in silica gels [21, 22]. These values are consistent with the $d=3$ connectivity percolation exponents measured here for attractive particulate systems, but are inconsistent with the connectivity percolation exponents that do not include interparticle forces.
Financial support from NSF grant numbers CTS0348175 (GL,JB) and DMR-0448838 (GL,CSO) is gratefully acknowledged. We also thank Yale's High Performance Computing Center for computing time.

[1] A. J. Liu and S. R. Nagel, Nature 396, 21 (1998);

[2] H. A. Makse, D. L. Johnson and L. M. Schwartz, Phys. Rev. Lett. 84, 4160 (2000); C. S. O'Hern, S. A. Langer, A. J. Liu and S. R. Nagel, Phys. Rev. Lett. 86, 111 (2001); C. S. O'Hern, L. E. Silbert, A. J. Liu and S. R. Nagel, Phys. Rev. E 68, 011306 (2003); H. P. Zhang and H. A. Makse, Phys. Rev. E 72, 011301 (2005). T. S. Majmudar, M. Sperl, S. Luding and R. P. Behringer, Phys. Rev. Lett. 98, 058001 (2007).

[3] N. Xu, M. Wyart, A. J. Liu and S. R. Nagel, Phys. Rev. Lett. 98, 175502 (2007).

[4] T. Eckert and E. Bartsch, Phys. Rev. Lett. 89, 125701 (2002); K. N. Pham et. al., Science 296, 104 (2002).

[5] J. M. Valverde, M. A. S. Quintanilla and A. Castellanos, Phys. Rev. Lett. 92, 258303 (2004); V. Richefeu, M. S. El Youssoufi and F. Radjai, Phys. Rev. E 73, 051304 (2006); F. A. Gilabert, J.-N. Roux and A. Castellanos, Phys. Rev. E 75, 011303 (2007).

[6] V. Trappe, V. Prasad, L. Cipelletti, P. N. Segre and D. A. Weitz, Nature 411, 772 (2001).

[7] S. Alexander, Phys. Rep. 296, 65 (1998).

[8] A. Tanguy, J. P. Wittmer, F. Leonforte and J.-L. Barrat, Phys. Rev. B 66, 174205 (2002).

[9] With periodic boundary conditions there are at least $d$ trivial zero eigenvalues, associated with translations of the entire system. In repulsive systems rattlers (particles with no contacts) also introduce trivial zero eigenvalues.

[10] "Floppers", which can involve many particles, are the generalization of rattlers in purely repulsive systems.

[11] D. Stauffer, Introduction to Percolation Theory (Taylor and Francis, London, 1985).

[12] E. T. Gawlinski and H. E. Stanley, J. Phys. A: Math. Gen. 14, L291 (1981).

[13] W. T. Elam, A. R. Kerstein and J. J. Rehr, Phys. Rev. Lett. 52, 1516 (1984).

[14] In bidisperse systems half of the particles are large and half are small, with diameter ratio 1.4 .

[15] Above $\phi_{\mathrm{R}}$, if a backbone exists then $P>0$. We have not been able to prove that $P>0$ implies the existence of a backbone since floppy modes might exist that are not excited by bulk compression. Our numerical results show that $P>0$ is equivalent to the existence of a backbone.

[16] D. J. Jacobs and M. F. Thorpe, Phys. Rev. Lett. 75, 4051 (1995); C. Moukarzel and P. M. Duxbury, Phys. Rev. Lett. 75, 4055 (1995).

[17] M. V. Chubynsky and M. F. Thorpe, to appear in Phys. Rev. E. (2007).

[18] F. Mallamace, P. Gambadauro, N. Micali, P. Tartaglia, C. Liao and S.-H. Chen, Phys. Rev. Lett. 84, 5431 (2000).

[19] G. Dietler, C. Aubert, D. S. Cannell and P. Wiltzius, Phys. Rev. Lett. 57, 3117 (1986).

[20] A. D. Dinsmore and D. A. Weitz, J. Phys.: Condens. Matter 14, 7581 (2002).

[21] J. E. Martin, J. Wilcoxon and D. Adolf, Phys. Rev. . 36, 1803 (1987).

[22] $\nu$ is the unswelled value [21] and $\tau$ was inferred from measurements of $\gamma$ and the hyperscaling relation. 